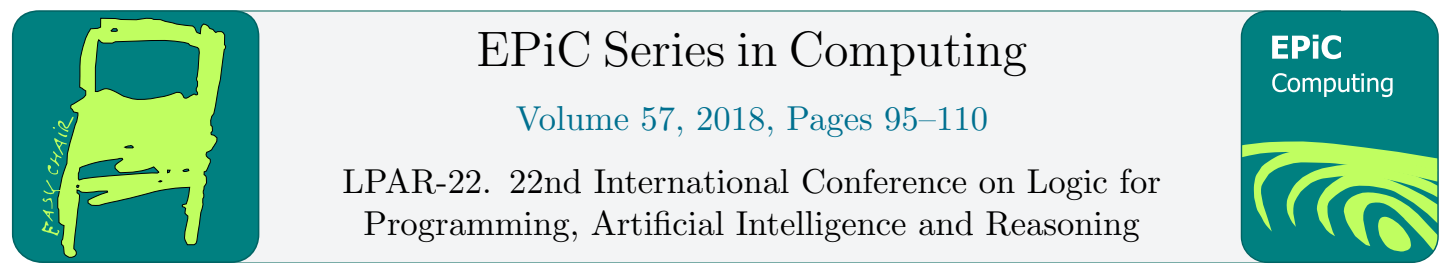

\title{
Lyndon Interpolation holds for the Prenex $\supset$ Prenex Fragment of Gödel Logic
}

\author{
Matthias Baaz ${ }^{1 *}$ and Anela Lolic ${ }^{2 \dagger}$ \\ 1 TU Wien, Institute of Discrete Mathematics and Geometry \\ baaz@logic .at \\ 2 TU Wien, Institute of Discrete Mathematics and Geometry \\ anela@logic.at
}

\begin{abstract}
First-order interpolation properties are notoriously hard to determine, even for logics where propositional interpolation is more or less obvious. One of the most prominent examples is first-order Gödel logic. Lyndon interpolation is a strengthening of the interpolation property in the sense that propositional variables or predicate symbols are only allowed to occur positively (negatively) in the interpolant if they occur positively (negatively) on both sides of the implication. Note that Lyndon interpolation is difficult to establish for first-order logics as most proof-theoretic methods fail. In this paper we provide general derivability conditions for a first-order logic to admit Lyndon interpolation for the prenex $\supset$ prenex fragment and apply the arguments to the prenex $\supset$ prenex fragment of first-order Gödel logic.
\end{abstract}

\section{Introduction}

The Craig interpolation property is one of the most fundamental properties of logics. It states that whenever $A \supset B$ is valid in a logic, one can find a formula $I$ in the common language of $A$ and $B$, such that $A \supset I$ and $I \supset B$ are valid. The formula $I$ is called the interpolant of $A \supset B$. Interpolation was proved for classical first-order logic by Craig [8] and for intuitionistic first-order logic by Schütte [17].

Lyndon interpolation is a strengthening of the interpolation property in the sense that propositional variables or predicate symbols are only allowed to occur positively (negatively) in the interpolant if they occur positively (negatively) on both sides of the implication. Lyndon interpolation for classical logic has been established by Lyndon [13].

Little was known about interpolation properties of intermediate logics until Maksimova solved the propositional interpolation problem showing that exactly 7 of these logics have the propositional interpolation property [14]. Her work is based on the algebraic analysis of the logic

\footnotetext{
${ }^{*}$ This work is partially supported by the FWF projects P 31063 and I 2671.

${ }^{\dagger}$ Recipient of a DOC Fellowship of the Austrian Academy of Sciences at the Institute of Logic and Computation, Theory and Logic Group, TU Wien, Austria.
}

G. Barthe, G. Sutcliffe and M. Veanes (eds.), LPAR-22 (EPiC Series in Computing, vol. 57), pp. 95-110 
in question. On first-order level, algebraic semantics are not as well understood and therefore first-order interpolation properties are notoriously hard to determine, even for logics where propositional interpolation is more of less obvious. Hence, it remained an open question which of these 7 logics admit first-order interpolation, among them first-order Gödel logic. Moreover, except of classical and intuitionistic logic, most logics do not seem to admit a Maehara style lemma w.r.t. their established calculi. This applies to e.g. all hypersequent calculi.

In [5] it has been shown that first-order finitely valued logics admit first-order interpolation if they admit propositional interpolation. In this paper we extend these results to the prenex $\supset$ prenex fragment of Gödel logic making use of the proof of Lyndon interpolation for propositional Gödel logic from [12]. Note that Lyndon interpolation is difficult to establish for first-order logics as most proof-theoretic methods fail.

\section{Abstract Conditions for Lyndon Interpolation}

In this section we provide general conditions for a first-order logic to admit Lyndon interpolation for the prenex $\supset$ prenex fragment.

Definition 1 (propositional and first-order language). A propositional language $L_{0}$ is given by countably many variables $x_{1}, x_{2}, \ldots$ and a set of connectives including $\wedge, \vee, \supset$ and is closed under application of the connectives.

A first-order language $L_{1}$ extending $L_{0}$ is given by countably many free variables, countably many bound variables, countably many function symbols of all finite arities, countably many predicate symbols of all finite arities and quantifiers $\forall$ and $\exists$. First-order expressions are predicates applied to terms (atomic expressions) and expressions obtained by closure under the connectives and the quantifiers $\forall$ and $\exists$ such that $\forall x A(x)(\exists x A(x))$ is an expression whenever $A(a)$ is an expression for a free variable $a$. logic.

In our context we presuppose notions of derivation $\vdash_{0}\left(\vdash_{1}\right)$ for propositional (first-order)

Definition 2 (propositional and first-order logic). Propositional and first-order logics are identified with $\left\{A \mid \vdash_{0} A\right\}$ and $\left\{A \mid \vdash_{1} A\right\}$ respectively. $A_{1}, \ldots, A_{n} \vdash_{0} B$ and $A_{1}, \ldots, A_{n} \vdash_{1} B$ denote $\vdash_{0} B$ whenever $\vdash_{0} A_{1}, \ldots, \vdash_{0} A_{n}$ and $\vdash_{1} B$ whenever $\vdash_{1} A_{1}, \ldots, \vdash_{1} A_{n} . \vdash A$ denotes $\vdash_{0} A$ and $\vdash_{1} A$.

The following definition represents the first $\varepsilon$-theorem in classical logic [11].

Definition 3 (extension and reduct). A first-order logic is called an extension of a propositional logic with the same connectives iff

1. $\vdash_{0} A$ implies $\vdash_{1} A^{\prime}$ for a substitution instance $A^{\prime}$ of $A$ by atomic expressions,

2. for quantifier-free $A, \vdash_{1} A$ implies that for some propositional $A^{\prime}, \vdash_{0} A^{\prime}$ and $A$ is a substitution instance of $A^{\prime}$ by atomic expressions.

The propositional logic is called the reduct of the first-order logic.

Interpolation is defined as usual and Lyndon interpolation as a refinement of usual interpolation.

Definition 4 (interpolation). A propositional or first-order logic admits interpolation if whenever $\vdash A \supset B$ there exists an interpolant $I$ such that $\vdash A \supset I$ and $\vdash I \supset B$ and $I$ is in the common language of $A$ and $B$. 
Definition 5 (Lyndon interpolation). A propositional interpolant $I$ of $\vdash A \supset B$ is called propositional Lyndon interpolant if every propositional variable occurring positively (negatively) in I occurs positively (negatively) in both $A$ and $B$. A first-order interpolant $I$ of $\vdash A \supset B$ is called first-order Lyndon interpolant if every predicate symbol occurring positively (negatively) in I occurs positively (negatively) in both $A$ and $B$.

The main arguments of this paper are based on the existence of suitable Skolemizations and Herbrand expansions. In our context universal quantifier occurrences left and existential quantifier occurrences right to the main implication of prenex $\supset$ prenex are called weak quantifier occurrences. Universal quantifier occurrences right and existential quantifier occurrences left to the main implication of prenex $\supset$ prenex are called strong quantifier occurrences.

Definition 6 (Skolemization). Let $F$ be a formula in prenex form. Its Skolemization is defined inductively via the function sk:

- If $F$ does not contain strong quantifiers, then $\operatorname{sk}(F)=F$.

- If $F=Q_{1} x_{1} \ldots Q_{n} x_{n} Q x F^{\prime}$, where $Q_{1}, \ldots, Q_{n}$ are weak quantifiers, $Q$ is the first occurrence of a strong quantifier and $F^{\prime}$ is a formula in prenex form, then $\operatorname{sk}\left(F^{\prime}\right)=$

$$
\operatorname{sk}\left(Q_{1} x_{1} \ldots Q_{n} x_{n} F^{\prime}\left\{x \leftarrow f\left(x_{1}, \ldots, x_{n}\right)\right\}\right),
$$

where $f$ is a fresh function symbol (a Skolem function).

We will Skolemize formulas $A \supset B, A, B$ prenex, thus obtaining a so-called Skolem form $(A \supset B)_{s}$.

Skolemized formulas contain at most weak quantifiers. These quantifiers can be eliminated by constructing so-called expansions. Expansions, first introduced in [15], are natural structures representing the instantiated variables for quantified formulas and record the substitutions for quantifiers in an effort to recover a sound proof of the original formulation of Herbrand's theorem. As we work with Skolemized formulas, we consider only expansions for formulas with weak quantifiers and consequently, the arguments are simplified.

Definition $7(\wedge / \vee$-compatible logic). A logic is $\wedge / \vee$-compatible iff

1. $A \vdash A^{*}$ and $A^{*} \vdash A$ if $A$ and $A^{*}$ are variants by applying commutativity, associativity and idempotence to $\wedge, \vee$,

2. $A \supset B \vdash A \wedge C \supset B$

3. $A \supset B \vdash A \supset B \vee C$

Definition 8 (expansion). Let an $\wedge / \vee$-compatible logic be given and let $\forall \vec{x} A(\vec{x}) \supset \exists \vec{y} B(\vec{y})$ be a formula, where $A$ and $B$ are quantifier free. An expansion of $\forall \vec{x} A(\vec{x}) \supset \exists \vec{y} B(\vec{y})$ is a quantifier-free formula

$$
\bigwedge_{i=1}^{n} A\left(\overrightarrow{s_{i}}\right) \supset \bigvee_{j=1}^{m} B\left(\overrightarrow{t_{j}}\right)
$$

which is obtained from $\forall \vec{x} A(\vec{x}) \supset \exists \vec{y} B(\vec{y})$ by constructing instances $A\left(\overrightarrow{s_{i}}\right), B\left(\overrightarrow{t_{j}}\right)$ for the quantified formulas by substituting quantified variables by suitable closed terms of the language of $\forall \vec{x} A(\vec{x}) \supset \exists \vec{y} B(\vec{y})$ (a constant is added if there is none). If an expansion is derivable, it is called 
a Herbrand expansion. Without loss of generality we may assume that Herbrand expansions are of the form

$$
\bigwedge_{i=1}^{n} A\left(\overrightarrow{s_{i}}\right) \supset \bigvee_{j=1}^{n} B\left(\overrightarrow{t_{j}}\right)
$$

(i.e. $m=n$ in the definition of expansions). A Herbrand expansion is a minimal Herbrand expansion of a formula $\forall \vec{x} A(\vec{x}) \supset \exists \vec{y} B(\vec{y})$ if $n$ is minimal. A logic admitting Herbrand expansions for formulas $\forall \vec{x} A(\vec{x}) \supset \exists \vec{y} B(\vec{y})$ is called weakly-expansive.

The following abstract conditions on logics w.r.t. derivability ensure a transfer of the propositional interpolation property to first-order.

Definition 9 (admissible logic). A weakly-expansive first-order logic $\vdash_{1}$ extending a propositional logic $\vdash_{0}$ is called admissible iff the following conditions hold:

1. $\vdash_{0} A \supset A$ for all propositional formulas $A$,

2.

$$
\begin{aligned}
& \left(\bigwedge A_{i} \supset \bigvee\left(B_{j} \vee C(a)\right)\right) \vdash_{1}\left(\bigwedge A_{i} \supset \bigvee\left(B_{j} \vee \forall x C(x)\right)\right) \\
& \left(\bigwedge A_{i} \supset \bigvee\left(B_{j} \vee C(t)\right)\right) \vdash_{1}\left(\bigwedge A_{i} \supset \bigvee\left(B_{j} \vee \exists x C(x)\right)\right) \\
& \left(\bigwedge\left(A_{i} \vee C(a)\right) \supset \bigvee B_{j}\right) \vdash_{1}\left(\bigwedge\left(A_{i} \vee \exists x C(x)\right) \supset \bigvee B_{j}\right) \\
& \left(\bigwedge\left(A_{i} \vee C(t)\right) \supset \bigvee B_{j}\right) \vdash_{1}\left(\bigwedge\left(A_{i} \vee \forall x C(x)\right) \supset \bigvee B_{j}\right)
\end{aligned}
$$

where $a$ is an eigenvariable,

3. $A \supset B, B \supset C \vdash A \supset C$.

Henceforth, we will only consider admissible logics, i.e. the use of $\vdash_{1}\left(\vdash_{0}\right)$ will indicate that the logic is admissible.

It can be shown that the Skolem form of a formula can always be derived from the formula itself, i.e. that the Skolem form is a logical consequence of the original formula.

Proposition 1. Let $A, B$ prenex and $(A \supset B)_{s}$ the Skolem form of $A \supset B$. Then $\vdash_{1}(A \supset B)_{s}$ if $\vdash_{1} A \supset B$.

Proof. Derive stepwise

$$
Q_{1} x_{1} \ldots Q_{n} x_{n} \forall x \exists y_{1} \ldots \exists y_{m} F \supset Q_{1} x_{1} \ldots Q_{n} x_{n} \exists y_{1} \ldots \exists y_{m} F\left\{x \leftarrow f\left(x_{1}, \ldots, x_{n}\right)\right\}
$$

from suitable instances of $A \supset A$ and replace strong quantifiers on the right side step by step using application of transitivity. (The analogous argument holds for the left side.)

Remark 1. Note that in usual logics such as classical logic, intuitionistic logic, Gödel logic etc. the Skolem functions can be directly introduced by substitutions without additional cuts.

The following theorem is a variant of the second $\varepsilon$-theorem [11]. Note that the property of weak-expandability is essential.

Theorem 1. Let $A, B$ prenex and $(A \supset B)_{s} \equiv \forall \vec{x} A(\vec{x}) \supset \exists \vec{y} B(\vec{y})$ the Skolem form of $A \supset B$. Then $\vdash_{1} A \supset B$ if $\vdash_{1}(A \supset B)_{s}$. 
Proof. Construct Herbrand expansions $A_{t_{1}} \wedge \ldots \wedge A_{t_{n}} \supset B_{s_{1}} \vee \ldots \vee B_{s_{n}}$ from the derivable Skolem form $\forall \vec{x} A(\vec{x}) \supset \exists \vec{y} B(\vec{y})$. Replace all Skolem terms $f\left(t_{1}, \ldots, t_{n}\right)$ not occurring in the scope of other Skolem functions by variables $a_{f\left(t_{1}, \ldots, t_{n}\right)}$. (A Skolem term is a term whose outermost symbol is a Skolem function symbol.) This replacement is sound as the first-order logic extends a propositional logic, cf. Definition 3. We use the following algorithm:

1. Introduce weak quantifiers according to $\forall \vec{x} A(\vec{x}) \supset \exists \vec{y} B(\vec{y})$ as often as possible and contract identical copies in the left or right side of the main implication

2. If 1 . is not possible and the original implication has not been obtained introduce a strong quantifier to the variable with the largest index still available. Go back to 1

Note that the inference in 2. fulfills the eigenvariable condition because otherwise either a variable with a larger Skolem term as index would be available or two identical copies would not have been contracted.

Example 1. Consider

$$
\vdash_{1} \forall x \exists y(C(x) \supset C(y)) \supset \exists x \forall y(C(x) \supset C(y))
$$

in classical logic. $\left(\vdash_{1} \forall x \exists y(C(x) \supset C(y)) \supset \exists x \forall y(C(x) \supset C(y))\right)_{s} \equiv \forall x(C(x) \supset C(f(x))) \supset$ $\exists x(C(x) \supset C(g(x)))$. As classical logic is weakly-expansive we obtain e.g. the Herbrand expansion

$$
\vdash_{1}(C(d) \supset C(f(d))) \wedge(C(d) \supset C(f(d))) \supset(C(d) \supset C(g(d)) \vee(C(g(d)) \supset C(g(g(d)))) .
$$

Replace Skolem terms $f(d), g(d), g(g(d))$ by variables $a_{f(d)}, a_{g(d)}, a_{g(g(d))}$

$$
\vdash_{1}\left(C(d) \supset C\left(a_{f(d)}\right)\right) \wedge\left(C(d) \supset C\left(a_{f(d)}\right)\right) \supset\left(C(d) \supset C\left(a_{g(d)}\right) \vee\left(C\left(a_{g(d)}\right) \supset C\left(a_{g(g(d))}\right)\right) .\right.
$$

Use the algorithm

1.

$$
\vdash_{1}\left(C(d) \supset C\left(a_{f(d)}\right)\right) \supset\left(C(d) \supset C\left(a_{g(d)}\right) \vee\left(C\left(a_{g(d)}\right) \supset C\left(a_{g(g(d))}\right)\right)\right.
$$

by $1: \wedge$-contraction

2.

$$
\vdash_{1}\left(C(d) \supset C\left(a_{f(d)}\right)\right) \supset\left(C(d) \supset C\left(a_{g(d)}\right) \vee \forall y\left(C\left(a_{g(d)}\right) \supset C(y)\right)\right.
$$

by $2: \forall$-introduction (alternatively $\exists$ could have been introduced on $\left.a_{f(d)}\right) . g(d)$ is subterm of $g(g(d))$.

3.

$$
\vdash_{1}\left(C(d) \supset C\left(a_{f(d)}\right)\right) \supset\left(C(d) \supset C\left(a_{g(d)}\right) \vee \exists x \forall y(C(x) \supset C(y))\right.
$$

by $1: \exists$-introduction

4.

$$
\vdash_{1} \exists y(C(d) \supset C(y)) \supset\left(C(d) \supset C\left(a_{g(d)}\right) \vee \exists x \forall y(C(x) \supset C(y))\right.
$$

by $2: \exists$-introduction (alternatively $\forall$ could have been introduced in $a_{g(d)}$ )

5.

$$
\vdash_{1} \forall x \exists y(C(x) \supset C(y)) \supset\left(C(d) \supset C\left(a_{g(d)}\right) \vee \exists x \forall y(C(x) \supset C(y))\right.
$$

by $1: \forall$-introduction 
6 .

$$
\vdash_{1} \forall x \exists y(C(x) \supset C(y)) \supset \forall y(C(d) \supset C(y) \vee \exists x \forall y(C(x) \supset C(y))
$$

by 2 : properties of $\vee, \forall$-introduction

7

$$
\vdash_{1} \forall x \exists y(C(x) \supset C(y)) \supset \exists x \forall y(C(x) \supset C(y) \vee \exists x \forall y(C(x) \supset C(y))
$$

by 1 : properties of $\vee, \exists$-introduction

8 .

$$
\vdash_{1} \forall x \exists y(C(x) \supset C(y)) \supset \exists x \forall y(C(x) \supset C(y))
$$

by $1: \vee$-contraction

Now we can prove that for admissible logics the propositional Lyndon interpolation property transfers to first-order.

Theorem 2. An admissible first-order logic admits Lyndon interpolation iff its propositional reduct does.

Proof. $\Rightarrow$ : obvious.

$\Leftarrow$ :

1. First Skolemize $A \supset B$. Let $\forall \vec{x} A(\vec{x}) \supset \exists \vec{y} B(\vec{y})$ be the result.

2. Derive a Herbrand expansion $A\left(\overrightarrow{t_{1}}\right) \wedge \ldots \wedge A\left(\overrightarrow{t_{n}}\right) \supset B\left(\overrightarrow{s_{1}}\right) \vee \ldots \vee B\left(\overrightarrow{s_{n}}\right)$.

3. Calculate a quantifier-free Lyndon interpolant $I$ such that

$$
\left.\vdash_{0} A\left(\overrightarrow{t_{1}}\right) \wedge \ldots \wedge A\left(\overrightarrow{t_{n}}\right)\right) \supset I \text { and } \quad \vdash_{0} I \supset B\left(\overrightarrow{s_{1}}\right) \vee \ldots \vee B\left(\overrightarrow{s_{n}}\right)
$$

Note that this construction is obtained in the following way: $A\left(\overrightarrow{t_{1}}\right) \wedge \ldots \wedge A\left(\overrightarrow{t_{n}}\right) \supset$ $B\left(\overrightarrow{s_{1}}\right) \vee \ldots \vee B\left(\overrightarrow{s_{n}}\right)$ is a substitution instance of some derivable propositional formula $A_{1}^{\prime} \wedge \ldots \wedge A_{n}^{\prime} \supset B_{1}^{\prime} \vee \ldots \vee B_{n}^{\prime}$ by replacing propositional variables by atomic expressions. We derive $A_{1}^{\prime} \wedge \ldots \wedge A_{n}^{\prime} \supset I^{\prime}$ and $I^{\prime} \supset B_{1}^{\prime} \vee \ldots \vee B_{n}^{\prime}$ for a propositional Lyndon interpolant $I^{\prime}$. Substitute the corresponding atomic expressions. The occurrence restrictions of propositional variables obviously transfer to the predicate symbols.

4. Partition the functions not in the common language of $\forall \vec{x} A(\vec{x})$ and $\exists \vec{y} B(\vec{y})$ (note that this includes the Skolem functions). Let $g_{1}, \ldots, g_{p}$ be the function symbols not occurring in the language of $\forall \vec{x} A(\vec{x})$ and $f_{1}, \ldots, f_{q}$ the function symbols not occurring in the language of $\exists \vec{y} B(\vec{y})$. Replace all terms $f_{i}\left(\overrightarrow{t_{j}}\right)$ not in the scope of other function symbols $f_{l}$ in

$$
I \supset B\left(\overrightarrow{s_{1}}\right) \vee \ldots \vee B\left(\overrightarrow{s_{n}}\right)
$$

by $a_{f_{i}\left(t_{j}\right)}$ and all terms $g_{i}\left(t_{j}\right)$ not in the scope of other function symbols $g_{k}$ in

$$
A\left(\overrightarrow{t_{1}}\right) \wedge \ldots \wedge A\left(\overrightarrow{t_{n}}\right) \supset I
$$

by $a_{g_{i}\left(t_{j}\right)}$. We obtain derivable formulas (same argument as in 3.)

$$
A\left(\overrightarrow{t_{1}^{\prime}}\right) \wedge \ldots \wedge A\left(\overrightarrow{t_{n}^{\prime}}\right) \supset I_{1} \quad \text { and } \quad I_{2} \supset B\left(\overrightarrow{s_{1}^{\prime}}\right) \vee \ldots \vee B\left(\overrightarrow{s_{n}^{\prime}}\right)
$$

5. Reintroduce $\forall$-left and $\exists$-right to obtain $\forall \vec{x} A(\vec{x}) \supset I_{1}$ and $I_{2} \supset \exists \vec{y} B(\vec{y})$. 
6. Order the indices of the introduced variables by inclusion. Introduce a strong quantifier for the variable with maximal index still available and dual a weak quantifier for the term at the same place of the free variable in the other occurrence of the potential interpolant. We obtain $\forall \vec{x} A(\vec{x}) \supset I^{\prime}$ and $I^{\prime} \supset \exists \vec{y} B(\vec{y})$.

7. Skolemize $I$ to obtain $\forall \vec{x} A(\vec{x}) \supset \exists x I_{1}^{*}$ and $\forall x I_{2}^{*} \supset \exists \vec{y} B(\vec{y})$.

8. Calculate Herbrand expansions and deskolemize according to Theorem 1 to obtain derivable formulas $A \supset I$ and $I \supset B$.

(7. can be avoided if instead of quantifiers suitable Skolem terms are substituted for the free variables in 6.).

Corollary 1. If an admissible first-order logic admits Lyndon interpolation for the prenex $\supset$ prenex fragment, then the interpolant is prenex.

Proof. By construction in the proof of Theorem 2.

The following example illustrated the procedure in the proof of Theorem 2.

Example 2. Consider $\forall x A(x, f(x)) \supset \exists y A(c, y)$ in classical logic, with a Herbrand expansion $A(c, f(c)) \supset A(c, f(c))$ (classical logic is obviously an admissible logic). $A(c, f(c))$ is a Lyndon interpolant of the Herbrand expansion, as

$$
\vdash_{1} A(c, f(c)) \supset A(c, f(c)) \quad \text { and } \quad \vdash_{1} A(c, f(c)) \supset A(c, f(c)) .
$$

We introduce variables $a_{c}$ and $b_{f(c)}$ for function terms not in the common language of $\forall x A(x, f(x))$ and $\exists y A(c, y)$. As $c$ occurs on the right side of $\forall x A(x, f(x)) \supset \exists y A(c, y)$ only, $a_{c}$ replaces $c$ everywhere in the left implication, $\vdash_{1} A(c, f(c)) \supset A(c, f(c))$. As $f$ occurs on the left side of $\forall x A(x, f(x)) \supset \exists y A(c, y)$ only, $b_{f(c)}$ replaces $f(c)$ everywhere in the right implication, $\vdash_{1} A(c, f(c)) \supset A(c, f(c))$. We obtain

$$
\vdash_{1} A\left(a_{c}, f\left(a_{c}\right)\right) \supset A\left(a_{c}, f\left(a_{c}\right)\right) \quad \text { and } \quad \vdash_{1} A\left(c, b_{f(c)}\right) \supset A\left(c, b_{f(c)}\right) .
$$

We introduce weak quantifiers and obtain

$$
\vdash_{1} \forall x A(x, f(x)) \supset A\left(a_{c}, f\left(a_{c}\right)\right) \quad \text { and } \quad \vdash_{1} A\left(c, b_{f(c)}\right) \supset \exists y A(c, y) .
$$

First we eliminate the variable with maximal index $b_{f(c)}$ and obtain

$$
\vdash_{1} \forall x A(x, f(x)) \supset \exists y A\left(a_{c}, y\right) \quad \text { and } \quad \vdash_{1} \exists y A(c, y) \supset \exists y A(c, y) .
$$

Then we eliminate $a_{c}$ and obtain

$$
\vdash_{1} \forall x A(x, f(x)) \supset \forall x \exists y A(x, y) \quad \text { and } \quad \vdash_{1} \forall x \exists y A(x, y) \supset \exists y A(c, y) .
$$

$\forall x \exists y A(x, y)$ is the Lyndon interpolant. Note that the introduction of the first strong quantifier for $a_{c}$ instead for $f\left(a_{c}\right)$ would render $f$ not eliminable. 


\section{Complexity of the First-Order Lyndon Interpolant}

We will use symbolic and logical complexity as complexity measures.

Definition 10 (symbolic complexity). The symbolic complexity $\|E\|$ of an expression $E$ is inductively defined as

1. $\|E\|=1$ if $E$ is a free or bound variable,

2. $\left\|f\left(E_{1}, \ldots, E_{n}\right)\right\|=\left\|E_{1}\right\|+\ldots+\left\|E_{n}\right\|+1$, where $f$ is a function symbol,

3. $\left\|P\left(E_{1}, \ldots, E_{n}\right)\right\|=\left\|E_{1}\right\|+\ldots+\left\|E_{n}\right\|+1$, where $P$ is a predicate symbol,

4. $\left\|E_{1} \circ E_{2}\right\|=\left\|E_{1}\right\|+\left\|E_{2}\right\|+1$, where $\circ \in\{\wedge, \vee, \supset\}$,

5. $\|\forall x E\|=\|\exists x E\|=\|E\|+1$.

Definition 11 (logical complexity). The logical complexity $|A|$ of a formula $A$ is inductively defined as

1. $|A|=1$ if $A$ is atomic,

2. $|A \circ B|=|A|+|B|+1$, where $\circ \in\{\wedge, \vee, \supset\}$,

3. $|\forall x A(x)|=|\exists x A(x)|=|A|+1$.

Definition 12 (expansion degree). Let $\forall \vec{x} A(\vec{x}) \supset \exists \vec{y} B(\vec{y})$, where $A$ and $B$ are quantifier-free and let

$$
\bigwedge_{i=1}^{n} A\left(\overrightarrow{s_{i}}\right) \supset \bigvee_{j=1}^{n} B\left(\overrightarrow{t_{j}}\right)
$$

be a corresponding Herbrand expansion minimal in $n$. Then the expansion degree is defined as

$$
\operatorname{ED}(\forall \vec{x} A(\vec{x}) \supset \exists \vec{y} B(\vec{y}))=n .
$$

In the proof of the main theorem of this section we will use unification.

Definition 13 (unification problem). A unification problem is defined as a set of equations $\mathcal{U}=\left\{h_{i} \equiv h_{i^{\prime}} \mid 1 \leq i \leq n\right\} . \mathcal{U}$ is solvable if there exists a substitution $\sigma$ such that $\left\{h_{i} \sigma \equiv\right.$ $\left.h_{i^{\prime}} \sigma \mid 1 \leq i \leq n\right\}=\left\{f_{i} \equiv f_{i} \mid 1 \leq i \leq n\right\} . \sigma$ is called a unifier of $\mathcal{U}$. A unifier $\sigma$ is called a most general unifier, if for any other unifier $\sigma^{\prime}$ of $\mathcal{U}$ there exists a substitution $\mu$ such that $\sigma^{\prime}=\sigma \mu$.

The symbolic complexity $\left\|\mathcal{U}_{\sigma}\right\|$ of $\mathcal{U}_{\sigma}=\left\{h_{i} \sigma \equiv h_{i}^{\prime} \sigma \mid 1 \leq i \leq n\right\}$ is defined as $\sum_{i=1}^{n}\left(\left\|h_{i} \sigma\right\|+\right.$ $\left.\| h_{i}^{\prime} \sigma||\right) .\|\mathcal{U}\|=\left\|\mathcal{U}_{\mathcal{E}}\right\|$ for the identity $\mathcal{E}$.

Proposition 2. Let $\sigma$ a most general unifier of a solvable unification problem $\mathcal{U}$. Then $\|\mathcal{U} \sigma\| \leq$ $\|\mathcal{U}\|^{2^{|| \mathcal{U}||}}$.

Proof. Note that by substituting a variable during the unification process the symbolic complexity gets at most squared. (The number of variable occurrences is bounded by the symbolic complexity). 
Theorem 3. Let $\operatorname{ED}(\forall \vec{x} A(\vec{x}) \supset \exists \vec{y} B(\vec{y}))=n$. There is a Herbrand expansion

$$
\bigwedge_{i=1}^{n} A\left(\overrightarrow{s_{i}^{\prime}}\right) \supset \bigvee_{j=1}^{n} B\left(\overrightarrow{t_{j}^{\prime}}\right)
$$

such that the symbolic complexity of $\bigwedge_{i=1}^{n} A\left(\overrightarrow{s_{i}^{\prime}}\right) \supset \bigvee_{j=1}^{n} B\left(\overrightarrow{t_{j}^{\prime}}\right)$ is bounded by

$$
(\|\forall \vec{x} A(\vec{x}) \supset \exists \vec{y} B(\vec{y})\|+2) * \operatorname{ED}(\forall \vec{x} A(\vec{x}) \supset \exists \vec{y} B(\vec{y}))^{2^{(\|\forall \vec{x} A(\vec{x}) \supset \exists \vec{y} B(\vec{y})\|+2) * \operatorname{ED}(\forall \vec{x} A(\vec{x}) \supset \exists \vec{y} B(\vec{y}))} .}
$$

Proof. We consider the following abstraction: new different free variables $\overrightarrow{a_{i}}, \overrightarrow{b_{j}}$ are associated with variables occurring in $\forall \vec{x} A(\vec{x}) \supset \exists \vec{y} B(\vec{y})$ and an expansion (not necessarily a Herbrand expansion)

$$
\bigwedge_{i=1}^{n} A\left(\overrightarrow{a_{i}}\right) \supset \bigvee_{j=1}^{n} B\left(\overrightarrow{b_{j}}\right)
$$

is obtained. By assumption, a Herbrand expansion

$$
\bigwedge_{i=1}^{n} A\left(\overrightarrow{s_{i}}\right) \supset \bigvee_{j=1}^{n} B\left(\overrightarrow{t_{j}}\right)
$$

exists. We consider the unification problem $\mathcal{U}=\left\{b \equiv b^{\prime}\right\}$, where $b$ and $b^{\prime}$ are atomic expressions in the abstract expansion $\bigwedge_{i=1}^{n} A\left(\overrightarrow{a_{i}}\right) \supset \bigvee_{j=1}^{n} B\left(\overrightarrow{b_{j}}\right)$ which correspond to identical atoms in $\bigwedge_{i=1}^{n} A\left(\overrightarrow{s_{i}}\right) \supset \bigvee_{j=1}^{n} B\left(\overrightarrow{t_{j}}\right)$. Let $\sigma$ be a most general unifier of $\mathcal{U}$. Then

$$
\bigwedge_{i=1}^{n} A\left(\overrightarrow{a_{i}}\right) \sigma \supset \bigvee_{j=1}^{n} B\left(\overrightarrow{b_{j}}\right) \sigma
$$

is a Herbrand expansion and its symbolic complexity is bounded by

$$
(\|\forall \vec{x} A(\vec{x}) \supset \exists \vec{y} B(\vec{y})\|+2) * \operatorname{ED}(\forall \vec{x} A(\vec{x}) \supset \exists \vec{y} B(\vec{y}))^{2^{(\|\forall \vec{x} A(\vec{x}) \supset \exists \vec{y} B(\vec{y})\|+2) * \operatorname{ED}(\forall \vec{x} A(\vec{x}) \supset \exists \vec{y} B(\vec{y}))} .}
$$

Corollary 2. The ED for derivable $\forall \vec{x} A(\vec{x}) \supset \exists \vec{y} B(\vec{y})$ can be calculated if the propositional reduct is decidable.

Proof. The procedure in the proof of Theorem 3 can be applied in all possible ways to $\bigwedge_{i=1}^{n} A\left(\overrightarrow{s_{i}}\right) \supset \bigvee_{j=1}^{n} B\left(\overrightarrow{t_{j}}\right)$ to determine whether there is a Herbrand expansion of this length.

The following example illustrates the procedure in the proof of Theorem 3.

Example 3. In all admissible logics $\vdash_{1} \forall x A(f(x)) \supset \exists y A(f(y))$. Note that $\| \forall x A(f(x)) \supset$ $\exists y A(f(y)) \|=9$ and $\operatorname{ED}(\forall x A(f(x)) \supset \exists y A(f(y)))=1$. Possible Herbrand expansions of $\forall x A(f(x)) \supset \exists y A(f(y))$ are

$$
\left.A\left(f^{n}(c)\right)\right) \supset A\left(f^{n}(c)\right), n \geq 1 .
$$

To generate an expansion with limited symbolic complexity we obtain, by associating a for $x$ and $b$ for $y$ in $\forall x A(f(x)) \supset \exists y A(f(y))$, the abstraction $A(f(a)) \supset A(f(b))$. As we know that $\left.A\left(f^{n}(c)\right)\right) \supset A\left(f^{n}(c)\right)$ is a Herbrand expansion we obtain $A(f(a)) \supset A(f(a))$ by unification. Note that $\|A(f(a)) \supset A(f(a))\|=7$. 
The complexity of a Lyndon interpolant is bounded by the complexity of the Herbrand expansion and the propositional interpolant.

Definition 14 (minimal logical complexity of a propositional Lyndon interpolant). $r(n)$ is the minimal logical complexity of a propositional Lyndon interpolant $I$ of $\vdash_{0} A \supset B$, whenever $|A| \leq n$ and $|B| \leq n$.

Theorem 4. For $\vdash_{1} A \supset B, A, B$ prenex, there is a Lyndon interpolant I with $\|I\| \leq$

$$
\begin{aligned}
& 2 *\left(\left\|(A \supset B)_{s}\right\|+2\right) * \operatorname{ED}\left((A \supset B)_{s}\right)^{2^{\left(\left\|(A \supset B)_{s}\right\|+2\right) * \operatorname{ED}\left((A \supset B)_{s}\right)}} \\
& * r\left(\left(\left\|(A \supset B)_{s}\right\|+2\right) * \operatorname{ED}\left((A \supset B)_{s}\right)^{\left.2^{\left(\left\|(A \supset B)_{s}\right\|+2\right) * \operatorname{ED}\left((A \supset B)_{s}\right)}\right),}\right.
\end{aligned}
$$

where $(A \supset B)_{s}$ is the Skolem form of $A \supset B$.

Proof. Note that the logical complexity is bounded by the symbolic complexity. Therefore there is a propositional interpolant bounded by

$$
r\left(\left(\left\|(A \supset B)_{s}\right\|+2\right) * \operatorname{ED}\left((A \supset B)_{s}\right)^{\left.2^{\left(\left\|(A \supset B)_{s}\right\|+2\right) * \operatorname{ED}\left((A \supset B)_{s}\right)}\right) .}\right.
$$

To obtain the symbolic complexity before introducing quantifiers in the interpolant we have to multiply wit

$$
2 *\left(\left\|(A \supset B)_{s}\right\|+2\right) * \operatorname{ED}\left((A \supset B)_{s}\right)^{2^{\left(\left\|(A \supset B)_{s}\right\|+2\right) * \operatorname{ED}\left((A \supset B)_{s}\right)} .}
$$

Furthermore, the symbolic complexity is doubled to cover the introduction of quantifiers in the interpolant.

\section{Lyndon Interpolation for Prenex $\supset$ Prenex in Gödel Logic}

Propositional finite-valued Gödel logics have been introduced by Gödel in 1933 [10] and later generalized by Dummett [9] to an infinite set of truth-values. The language of Gödel logics is identical to that of classical logic. We use the binary connectives $\wedge, \vee$ and $\supset$ and the truth constant $\perp$. $\neg A$ is defined as $A \supset \perp$.

Gödel logics can be identified with a countable family of many-valued logics where the set of truth values $V$ are closed sets $\{0,1\} \subseteq V \subseteq[0,1]$. Their connectives can be interpreted as functions over either $\left\{0, \frac{1}{k}, \ldots, \frac{k-1}{k}, 1\right\}$ (for $G_{k+1}$ ) or the real interval $[0,1]$ for $G_{[0,1]}$. We will consider the first-order Gödel logic $G_{[0,1]}$. An interpretation $\mathcal{I}$ in $G_{[0,1]}$ consists of a non-empty domain $D$ and a valuation function $\nu_{\mathcal{I}}$ that maps constants and object variables to elements of $D$ and $n$-ary function symbols to functions from $D^{n}$ to $D$. $\nu_{\mathcal{I}}$ extends to functions mapping all terms of the language to an element of the domain in the usual way. $\nu_{\mathcal{I}}$ maps every $n$ ary predicate symbol $P$ to a function from $D^{n}$ to $V$. The truth-value of an atomic formula $A=P\left(t_{1}, \ldots, t_{n}\right)$ is defined as

$$
\nu_{\mathcal{I}}(A)=\nu_{\mathcal{I}}(P)\left(\nu_{\mathcal{I}}\left(t_{1}\right), \ldots, \nu_{\mathcal{I}}\left(t_{n}\right)\right) .
$$

We define $\nu_{\mathcal{I}}(\perp)=0$.

The semantics of propositional connectives is given by

$$
\begin{aligned}
& \nu_{\mathcal{I}}(A \wedge B)=\quad \min \left(\nu_{\mathcal{I}}(A), \nu_{\mathcal{I}}(B)\right), \quad \nu_{\mathcal{I}}(A \vee B)=\max \left(\nu_{\mathcal{I}}(A), \nu_{\mathcal{I}}(B)\right), \\
& \nu_{\mathcal{I}}(A \supset B)= \begin{cases}1 & \text { if } \nu_{\mathcal{I}}(A) \leq \nu_{\mathcal{I}}(B) \\
\nu_{\mathcal{I}}(B) & \text { otherwise }\end{cases}
\end{aligned}
$$


In order to obtain a concise formulation of the semantics of quantifiers we define the distribution of a formula $A$ and a free variable $x$ with respect to an interpretation $\mathcal{I}$ as $\operatorname{Distr}_{\mathcal{I}}(A(x))=$ $\left\{\operatorname{val}_{\mathcal{I}^{\prime}}(A(x)) \mid \mathcal{I}^{\prime} \sim_{x} \mathcal{I}\right\}$, where $\mathcal{I}^{\prime} \sim_{x} \mathcal{I}$ means that $\mathcal{I}^{\prime}$ is exactly as $\mathcal{I}$ with the possible exception of the domain element assigned to $x$. The semantics of quantifiers is then defined as

$$
\nu_{\mathcal{I}}(\forall x A(x))=\inf \left(\operatorname{Distr}_{\mathcal{I}}(A(x))\right), \quad \nu_{\mathcal{I}}(\exists x A(x))=\sup \left(\operatorname{Distr}_{\mathcal{I}}(A(x))\right) .
$$

A formula $A$ is tautological iff for all $\nu_{\mathcal{I}}, \nu_{\mathcal{I}}(A)=1 . A$ is a logical consequence of a set of formulas $\Gamma, \Gamma \models A$, iff, for all $\nu_{\mathcal{I}}, \min \left\{\nu_{\mathcal{I}}(\gamma) \mid \gamma \in \Gamma\right\} \leq \nu_{\mathcal{I}}(A)$.

An analytic calculus for $G_{[0,1]}$ has been introduced in [7] and uses hypersequents, a natural generalization of Gentzen sequents (see [1]). Recall that a sequent is an expression of the form $\Gamma \rightarrow A$, where $\Gamma$ is a set of formulas and $A$ may be empty. A hypersequent is then defined as a set of components, where each component is a sequent.

Definition 15 (hypersequent). A hypersequent is a set

$$
\Gamma_{1} \rightarrow A_{1}|\ldots| \Gamma_{n} \rightarrow A_{n}
$$

where for every $i=1, \ldots, n, \Gamma_{i} \rightarrow A_{i}$ is a sequent, called component of the hypersequent.

We define a hypersequent calculus HIF $^{\text {set }}$, see Table 1, where the interpretation of the symbol $\mid$ is $\cup$. Henceforth, we will denote with $\left\{S_{1}\right\} \cup \ldots \cup\left\{S_{n}\right\}$ a hypersequent whose components are $S_{1}, \ldots, S_{n}$. The hypersequents-as-sets approach allows for more explicit bounds of cut-free proofs using a Schütte Tait style elimination procedure. For the relation to more conventional notions for hypersequents cf. [2,3].

Theorem 5. HIF ${ }^{\mathrm{set}}$ is sound and complete. Note that in $\mathbf{H I F}^{\mathrm{set}}$ non-atomic axioms can be derived from atomic axioms.

Proof. See e.g. [3].

$\vdash_{1} A$ in $G_{[0,1]}$ is represented by an end-hypersequent $\{\rightarrow A\}$ in HIF $^{\text {set }}$.

Definition 16 (derivation length). The length $|d|$ of a derivation $d$ in $\mathbf{H I F}^{\text {set }}$ is the maximal number of inference rules (but weakenings) +1 occurring on any branch of $d$.

The right (left) rank of a cut is the number of consecutive hypersequents containing the cut formula, counting upwards from the right (left) upper sequent of the cut.

Definition 17 (cut-rank). Let $d_{i}$, where $i<k$, be the direct subderivations of $d$. The cut-rank $\rho(d)$ of $d$ is defined as

1. $\rho(d)=0$ if $d$ is cut-free,

2. $\rho(d)=\max _{i<k} \rho\left(d_{i}\right)$ if the last inference of $d$ is not a cut,

3. $\rho(d)=\max \left(|A|+1, \max _{i<k} \rho\left(d_{i}\right)\right)$, where $A$ is the cut formula.

We will write $d \vdash H$ if $d$ is a derivation in $\mathbf{H I F}^{\text {set }}$ of $H$.

Theorem 6 (cut-elimination). If $d \vdash H$ and $\rho(d)>0$ then we can find a derivation $d^{\prime} \vdash H$ with $\rho\left(d^{\prime}\right)<\rho(d)$ and $\left|d^{\prime}\right| \leq 4^{|d|}$.

Proof. See proof of Theorem 1 in [2]. 
Axioms

$A \rightarrow A \quad \perp \rightarrow$

$A$ is atomic

External Structural Rules

$\frac{G}{G \mid \Gamma \rightarrow A}(e w)$

Internal Structural Rules

$\frac{G \mid \Gamma \rightarrow C}{G \mid \Gamma, A \rightarrow C}(w, l)$

$\frac{G \mid \Gamma \rightarrow}{G \mid \Gamma \rightarrow C}(w, r)$

Logical Rules

$\frac{G \mid \Gamma, A \rightarrow B}{G \mid \Gamma \rightarrow A \supset B}(\supset, r)$

$\frac{G\left|\Gamma \rightarrow A \quad G^{\prime}\right| \Gamma \rightarrow B}{G\left|G^{\prime}\right| \Gamma \rightarrow A \wedge B}(\wedge, r)$

$\frac{G \mid \Gamma \rightarrow A_{i}}{G \mid \Gamma \rightarrow A_{1} \vee A_{2}}\left(\vee_{i}, r\right)_{i=1,2}$

$\frac{G\left|\Gamma, \Gamma^{\prime} \rightarrow A \quad G^{\prime}\right| \Gamma_{1}, \Gamma_{1}^{\prime} \rightarrow A^{\prime}}{G\left|G^{\prime}\right| \Gamma, \Gamma_{1}^{\prime} \rightarrow A \mid \Gamma^{\prime}, \Gamma_{1} \rightarrow A^{\prime}}(\mathrm{com})$
Cut Rule

$\frac{G\left|\Gamma^{\prime} \rightarrow A \quad G^{\prime}\right| A, \Gamma \rightarrow C}{G\left|G^{\prime}\right| \Gamma, \Gamma^{\prime} \rightarrow C}($ cut $)$

Table 1: Hypersequent calculus $\mathbf{H I F}^{\text {set }}$

Corollary 3. If $d \vdash H$, one can find a cut-free proof $d^{\prime} \vdash H$ with $\left|d^{\prime}\right| \leq 4_{\rho(d)}^{|d|}$.

Proof. Follows directly from Theorem 6.

Theorem 7. The prenex $\supset$ prenex fragment of $G_{[0,1]}$ admits Lyndon interpolation.

Proof. All conditions for admissibility except the weak-expandability are obviously fulfilled by $G_{[0,1]}$. Weak-expandability follows by first eliminating cuts of a proof of $\rightarrow \forall \vec{x} A(\vec{x}) \supset \exists \vec{y} B(\vec{y})$ and then deleting the inferences of $\forall$ and $\exists$ and avoiding contractions and the final $(\supset, r)$ introduction. The only problematic step is the adaption of $(\vee, l)$. Transform such inferences to

$$
\begin{gathered}
\frac{G\left|\Gamma, A \rightarrow \bigvee C(t) \quad G^{\prime}\right| \Gamma, B \rightarrow \bigvee C\left(t^{\prime}\right)}{G\left|\Gamma, A \rightarrow \bigvee C(t) \vee \bigvee C\left(t^{\prime}\right)\right| G^{\prime} \mid \Gamma, B \rightarrow \bigvee C(t) \vee \bigvee C\left(t^{\prime}\right)} \\
\hline G\left|G^{\prime}\right| \Gamma, A \vee B \rightarrow \bigvee C(t) \vee \bigvee C\left(t^{\prime}\right)
\end{gathered}
$$


The result is a hypersequent

$$
A\left(t_{1_{1}}\right), \ldots, A\left(t_{1_{n_{1}}}\right) \rightarrow B\left(s_{1}\right) \vee \ldots \vee B\left(s_{1_{m_{1}}}\right)|\ldots| A\left(t_{r_{1}}\right), \ldots, A\left(t_{r_{n_{r}}}\right) \rightarrow B\left(s_{r}\right) \vee \ldots \vee B\left(s_{r_{m_{r}}}\right) .
$$

From this hypersequent we can easily derive

$$
\rightarrow \bigwedge A\left(\overrightarrow{t_{i}}\right) \supset \bigvee B\left(\overrightarrow{s_{j}}\right)
$$

$\operatorname{ED}(\forall \vec{x} A(\vec{x}) \supset \exists \vec{y} B(\vec{y}))$ is elementary in the maximal logical complexity of a cut, which follows by the bound of cut-elimination in Corollary 3. Note that propositional Gödel logic admits elementary Lyndon interpolation by Kuznets and Lellmann [12].

Corollary 4. The symbolic complexity of a first-order Lyndon interpolant of $A \supset B$ valid in $G_{[0,1]}$ can be bounded elementarily in the symbolic complexity of the end-formula and the logical complexity of the maximal cut.

Proof. $r$ is elementary by the construction in [12]. The cut rank of a proof is bounded by $|c|+1$ for a maximal cut $c . \operatorname{ED}\left((A \supset B)_{s}\right)$, where $(A \supset B)_{s}$ is the Skolem form of $A \supset B$, is obviously elementarily bounded in the length of the cut-free proof. (Skolemization in Gödel logics does not introduce new cuts.)

Example 4. Consider $\vdash_{1} \forall x(A(x) \wedge R(x)) \supset \forall y \forall z \exists u(B(u) \vee(B(z) \supset A(y)) \vee \neg R(y))$ in $G_{[0,1]}$ by the following derivation in $\mathbf{H I F}^{\mathrm{set}}$.

$$
\begin{gathered}
\frac{A(a) \rightarrow A(a) \quad B(b) \rightarrow B(b)}{A(a) \rightarrow B(b) \mid B(b) \rightarrow A(a)} \\
\frac{\frac{A(a) \rightarrow B(b) \mid \rightarrow B(b) \supset A(a)}{A(a) \rightarrow B(b) \mid \rightarrow B(b) \vee(B(b) \supset A(a))}}{\frac{A(a) \rightarrow B(b) \mid \rightarrow B(b) \vee(B(b) \supset A(a)) \vee \neg R(a)}{A(a) \rightarrow B(b) \mid A(a) \rightarrow B(b) \vee(B(b) \supset A(a)) \vee \neg R(a)}} \\
\frac{\frac{A(a) \rightarrow B(b) \vee(B(b) \supset A(a)) \vee \neg R(a)}{A(a) \wedge R(a) \rightarrow B(b) \vee(B(b) \supset A(a)) \vee \neg R(a)}}{\frac{\forall x(A(x) \wedge R(x)) \rightarrow B(b) \vee(B(b) \supset A(a)) \vee \neg R(a)}{\forall x(A(x) \wedge R(x)) \rightarrow \exists u(B(u) \vee(B(b) \supset A(a)) \vee \neg R(a))}} \\
\frac{\forall x(A(x) \wedge R(x)) \rightarrow \forall z \exists u(B(u) \vee(B(z) \supset A(a)) \vee \neg R(a))}{\forall x(A(x) \wedge R(x)) \rightarrow \forall y \forall z \exists u(B(u) \vee(B(z) \supset A(y)) \vee \neg R(y))} \\
\rightarrow \forall x(A(x) \wedge R(x)) \supset \forall y \forall z \exists u(B(u) \vee(B(z) \supset A(y)) \vee \neg R(y))
\end{gathered}
$$

$(\forall x(A(x) \wedge R(x)) \supset \forall y \forall z \exists u(B(u) \vee(B(z) \supset A(y)) \vee \neg R(y)))_{s} \equiv \forall x(A(x) \wedge R(x)) \supset \exists u(B(u) \vee$ $(B(g) \supset A(f)) \vee \neg R(f))$, where $f$ and $g$ are Skolem constants.

To obtain a derivation of the Skolemized formula we replace a everywhere by a Skolem term $f, b$ everywhere by a Skolem term $g$ : 


$$
\begin{gathered}
\frac{A(f) \rightarrow A(f) \quad B(g) \rightarrow B(g)}{A(f) \rightarrow B(g) \mid B(g) \rightarrow A(f)} \\
\frac{\frac{A(f) \rightarrow B(g) \mid \rightarrow B(g) \supset A(f)}{A(f) \rightarrow B(g) \mid \rightarrow B(g) \vee(B(g) \supset A(f))}}{\frac{A(f) \rightarrow B(g) \mid \rightarrow B(g) \vee(B(g) \supset A(f)) \vee \neg R(f)}{A(f) \rightarrow B(g) \mid A(f) \rightarrow B(g) \vee(B(g) \supset A(f)) \vee \neg R(f)}} \\
\frac{\frac{\Delta(g) \vee(B(g) \supset A(f)) \mid A(f) \rightarrow B(g) \vee(B(g) \supset A(f)) \vee \neg R(f)}{A(f) \wedge R(f) \rightarrow B(g) \vee(B(g) \supset A(f)) \vee \neg R(f)}}{\frac{\forall x(A(x) \wedge R(x)) \rightarrow B(g) \vee(B(g) \supset A(f)) \vee \neg R(f)}{\forall x(A(x) \wedge R(x)) \rightarrow \exists u(B(u) \vee(B(g) \supset A(f)) \vee \neg R(f))}}
\end{gathered}
$$

$(A(f) \wedge R(f)) \supset(B(g) \vee(B(g) \supset A(f)) \vee \neg R(f))$ is the Herbrand expansion obtained and $A(f)$ is the corresponding Lyndon interpolant $(R(f)$ does not occur).

Replace $f$ and $g$ in $A(f) \wedge R(f) \supset A(f)$ everywhere by new variables $c_{f}$ and $c_{g}$ to obtain

$$
A\left(c_{f}\right) \wedge R\left(c_{f}\right) \supset A\left(c_{f}\right) \quad A(f) \supset B(g) \vee(B(f) \supset A(f)) \vee \neg R(f) .
$$

Introduce weak quantifiers to obtain

$$
\forall x(A(x) \wedge R(x)) \supset A\left(c_{f}\right) \quad A(f) \supset \exists u(B(u) \vee(B(f) \supset A(f)) \vee \neg R(f)) .
$$

Introduce a strong quantifier $\forall$ and dual a weak quantifier $\forall$ on the potential interpolant

$$
\forall x(A(x) \wedge R(x)) \supset \forall x A(x) \quad \forall x A(x) \supset \exists u(B(u) \vee(B(f) \supset A(f)) \vee \neg R(f))
$$

and deskolemize. $\forall x A(x)$ is the constructed Lyndon interpolant.

The next example is a prenex variant of the counterexample $A \supset B$ to interpolation for constant domain intuitionistic logic by Mints, Olkhovikov and Urquhart [16].

Example 5. Let

$$
\begin{gathered}
A \equiv \forall x \exists y(P(y) \wedge(Q(y) \supset R(x))) \wedge \neg \forall x R(x) \text { and } \\
B \equiv \forall x(P(x) \supset(Q(x) \vee S)) \supset S .
\end{gathered}
$$

$A \supset B$ is valid in $G_{[0,1]}$ but does not provide a counterexample to interpolation and admits e.g. interpolants

$$
\begin{gathered}
I_{1}=\neg \forall x \neg \neg Q(x) \wedge \forall x \exists y(P(y) \wedge(Q(y) \rightarrow(Q(x) \vee \neg Q(x))) \wedge \neg \forall x(Q(x) \vee \neg Q(x))) \vee \\
\neg \neg \forall x \neg \neg Q(x) \wedge \forall x \exists y(P(y) \wedge(Q(y) \rightarrow Q(x))) \wedge \neg \forall x Q(x)
\end{gathered}
$$

or $I_{2}=\forall x(\neg P(x) \vee \exists y(P(y) \wedge(Q(y) \supset P(x)))) \wedge \neg \forall x(\neg P(x) \vee Q(x))$ [4].

Now consider $A^{\prime} \supset B^{\prime}$ with

$$
\begin{gathered}
A^{\prime} \equiv \exists u \forall x \exists y(P(y) \wedge(Q(y) \supset R(x)) \wedge \neg R(u)) \text { and } \\
B^{\prime} \equiv \exists x((P(x) \supset(Q(x) \vee S)) \supset X \vee X \supset S) .
\end{gathered}
$$


Then obviously $A^{\prime} \supset A$ as $A^{\prime}$ is equivalent to $\forall x \exists y(P(y) \wedge(Q(y) \supset R(x)) \wedge \exists x \neg R(x))$ and $B \supset B^{\prime}$ as $(\forall x E(x) \supset G) \supset \exists x(E(x) \supset H \vee H \supset G)$ is valid in $G_{[0,1]}$.

$$
\left(A^{\prime} \supset B^{\prime}\right)_{s} \equiv \forall x\left(P(f(x)) \wedge(Q(f(x)) \supset R(x)) \wedge \neg R(y) \supset B^{\prime} .\right.
$$

We guess a Herbrand expansion

$$
(P(f(g)) \wedge(Q(f(g)) \supset R(g)) \wedge \neg R(g)) \supset((P(f(g)) \supset(Q(f(g)) \vee S)) \supset X \vee X \supset S) .
$$

$P(f(g)) \wedge \neg Q(f(g))$ is a Lyndon interpolant for propositional logic, as the following implications are valid

$$
\begin{gathered}
(P(f(g)) \wedge(Q(f(g)) \supset R(g)) \wedge \neg R(g)) \supset(P(f(g)) \wedge \neg Q(f(g))), \\
(P(f(g)) \wedge \neg Q(f(g))) \supset((P(f(g)) \supset(Q(f(g)) \vee S)) \supset X \vee X \supset S) .
\end{gathered}
$$

Replace the only Skolem term $f(g)$ in the second implication everywhere by $a_{f(g)}$ to obtain

$$
\begin{gathered}
(P(f(g)) \wedge(Q(f(g)) \supset R(g)) \wedge \neg R(g)) \supset(P(f(g)) \wedge \neg Q(f(g))), \\
\left(P\left(a_{f(g)}\right) \wedge \neg Q\left(a_{f(g)}\right)\right) \supset\left(\left(P\left(a_{f(g)}\right) \supset\left(Q\left(a_{f(g)}\right) \vee S\right)\right) \supset X \vee X \supset S\right) .
\end{gathered}
$$

Introduce weak quantifiers to obtain

$$
\begin{gathered}
\forall x(P(f(x)) \wedge(Q(f(x)) \supset R(x)) \wedge \neg R(g)) \supset(P(f(g)) \wedge \neg Q(f(g))), \\
\left(P\left(a_{f(g)}\right) \wedge \neg Q\left(a_{f(g)}\right)\right) \supset \exists x((P(x) \supset(Q(x) \vee S)) \supset X \vee X \supset S) .
\end{gathered}
$$

Introduce a strong $\exists$ quantifier and a dual weak $\exists$ quantifier to the potential interpolant and deskolemize. The first-order Lyndon interpolant obtained is $\exists x(P(x) \wedge \neg Q(x))$.

By the Takeuti-Titani rule [18] this is also a first-order Lyndon interpolant for $A^{\prime} \supset B$ :

$$
\exists x((P(x) \supset Q(x) \vee S) \supset X \vee X \supset S) \supset \forall x(P(x) \supset Q(x) \vee S) \supset X \vee X \supset S
$$

is valid as a counterexample to $E \supset(F \supset S)$ implies the existence of a counterexample for $E \supset(F \supset X \vee X \supset S)$ if $X$ does not occur in $E, F, S$.

\section{Limits of the Method}

The results of this paper can be extended to other suitable refinements of propositional interpolation and provide first-order interpolants with the same refinements. It is however not the case that the constructions can be used to prove Lyndon interpolation or other forms of interpolation for full first-order Gödel logic as the interpolants constructed by our method are always prenex, see Corollary 1, and full first-order Gödel logic does not always admit prenex interpolants. Consider

$$
(C \wedge \neg \forall x P(x) \wedge \forall x \neg \neg P(x)) \supset(D \vee \neg \forall x P(x) \wedge \forall x \neg \neg P(x)) .
$$

Assume there is a prenex interpolant $I$. Then $G_{[0,1]} \models \neg \forall x P(x) \wedge \forall x \neg \neg P(x) \leftrightarrow I$ (replace $C, D$ by $\neg \forall x P(x) \wedge \forall x \neg \neg P(x))$. Consequently $G_{E} \models \neg \forall x P(x) \wedge \forall x \neg \neg P(x) \leftrightarrow I$ for all $E$ closed $\{0,1\} \subseteq E \subseteq[0,1] . \neg \forall x P(x) \wedge \forall x \neg \neg P(x)$ is 1-satisfiable in $G_{[0,1]}$ and so is $I . \neg \forall x P(x) \wedge$ $\forall x \neg \neg P(x)$ is not 1-satisfiable in $G_{\{0\} \cup\left[\frac{1}{2}, 1\right]}$. To obtain a contradiction we use the following proposition.

Proposition 3. A prenex formula is 1-satisfiable in any $G_{D} D$ closed $\{0,1\} \subseteq D \subseteq[0,1]$ iff it is 1-satisfiable in classical logic.

Proof. Cf. proof of Corollary 41 in [6]. 


\section{References}

[1] Arnon Avron. The method of hypersequents in the proof theory of propositional non-classical logics. Logic: from Foundations to Applications. European Logic Colloquium Oxford Science Publications. Clarendon Press, 1996.

[2] Matthias Baaz and Agata Ciabattoni. A Schütte-Tait style cut-elimination proof for first-order Gödel logic. In TABLEAUX, volume 2381 of Lecture Notes in Computer Science, pages 24-37. Springer, 2002.

[3] Matthias Baaz, Agata Ciabattoni, and Christian G. Fermüller. Hypersequent calculi for Gödel logics - a survey. J. Log. Comput., 13(6):835-861, 2003.

[4] Matthias Baaz, Mai Gehrke, and Sam van Gool. An interpolant in predicate Gödel logic. CoRR abs/1803.03003, 2018.

[5] Matthias Baaz and Anela Lolic. First-order interpolation of non-classical logics derived from propositional interpolation. In FroCoS, volume 10483 of Lecture Notes in Computer Science, pages 265-280. Springer, 2017.

[6] Matthias Baaz and Norbert Preining. On the classification of first order Gödel logics. Annals of Pure and Applied Logic, to appear, 2018.

[7] Matthias Baaz and Richard Zach. Hypersequent and the proof theory of intuitionistic fuzzy logic. In CSL, volume 1862 of Lecture Notes in Computer Science, pages 187-201. Springer, 2000.

[8] William Craig. Three uses of the Herbrand-Gentzen theorem in relating model theory and proof theory. The Journal of Symbolic Logic, 22(3):269-285, 1957.

[9] Michael Dummett. A propositional calculus with denumerable matrix. J. Symb. Log., 24(2):97106, 1959.

[10] Kurt Gödel. Zum intuitionistischen Aussagenkalkül. Ergebnisse eines mathematischen Kolloquiums, 4:34-38, 1933.

[11] David Hilbert and Paul Bernays. Grundlagen der Mathematik. 2, 1939.

[12] Roman Kuznets and Björn Lellmann. Interpolation for intermediate logics via hyper- and linear nested sequents. In AiML 2018. College Publications, 2018. To appear.

[13] Roger C. Lyndon. An interpolation theorem in the predicate calculus. Pacific J. Math., 9(1):129142, 1959.

[14] LL Maksimova. Craig's interpolation theorem and amalgamated varieties of pseudoboolean algebras. Algebra and Logic, 16:643-681, 1977.

[15] Dale A Miller. A compact representation of proofs. Studia Logica, 46(4):347-370, 1987.

[16] Grigori Mints, Grigory Olkhovikov, and Alasdair Urquhart. Failure of interpolation in constant domain intuitionistic logic. The Journal of Symbolic Logic, 78(3):937-950, 2013.

[17] Kurt Schütte. Der Interpolationssatz der intuitionistischen Prädikatenlogik. Mathematische Annalen, 148(3):192-200, 1962.

[18] Gaisi Takeuti and Satoko Titani. Intuitionistic fuzzy logic and intuitionistic fuzzy set theory. The Journal of Symbolic Logic, 49(3):851-866, 1984. 